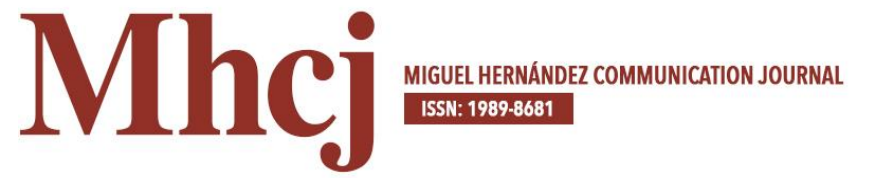

\title{
Los estudios cinematográficos en Chile: aproximaciones a la conformación y reflexión de campo
}

\author{
María Marcela Parada Poblete| mparadap@uc.cl \\ Pontificia Universidad Católica de Chile
}

\section{Palabras clave}

Cine chileno; Estudios en cine; Campo

cultural.

\section{Sumario}

1. Introducción. 2. Metodología 3. Resultados

4. Discusión 5. Conclusiones. 6. Bibliografía.

\section{Resumen}

Este trabajo comparte el estado de avance de la investigación "Mapa de los estudios de cine en Chile (2005-2015)", proyecto financiado por el Fondo de Fomento Audiovisual de Chile convocatoria 2016. Interesa dar cuenta del panorama de campo que se ha enfrentado, así como del proceso de construcción de una matriz de análisis para

los textos en estudio en el período señalado, incluyendo los principales resultados hallados en el trayecto de la investigación en curso. En cuanto a la cartografía de los estudios publicados en Chile como libros, capítulos de libro y artículos en revistas académicas en la década en revisión, la investigación arrojó un corpus total de 452 publicaciones. La metodología de análisis se aborda a partir de una matriz de lectura y vaciado de textos realizada específicamente para esta investigación cuyo diseño preliminar, sometido a discusión de expertos nacionales e internacionales, orientó respecto de coincidencias y discordancias en relación a las premisas con las cuales se inició este proyecto y señaló la ruta para acotar el instrumento de análisis, a la fecha (octubre del 2016) en aplicación. Esta investigación proyecta aspectos metodológicos y prácticos que contribuyen al desarrollo y discusión de los estudios de cine en Chile y Latinoamérica.

\section{Cómo citar este texto:}

María Marcela Parada Poblete (2017): “ Los estudios cinematográficos en Chile: aproximaciones a la conformación y reflexión de campo", en Miguel Hernández Communication Journal, nº, pp. 85 a 119. Universidad Miguel Hernández, UMH (ElcheAlicante). Recuperado el _ de de 20 de: [link del artículo en mhjournal.org] 


\title{
The film studies in Chile: an approach to the configuration and thinking of a field
}

\author{
María Marcela Parada Poblete| mparadap@uc.cl \\ Pontificia Universidad Católica de Chile
}

\section{Keywords}

Cinema Studies, Mexico, 21st Century, Film

Analysis, Lauro Zavala, Film Theory

Summary

1. Introduction. 2. Methodology. 3. Results. 4.

Discussion. 5. Conclusions. 6. Bibliography.

\section{Abstract}

This work shares the progress of the investigation "Maps of the films studies in Chile (2005-2015)", project funded by the Chilean Audiovisual Development Fund 2016. It is interested in revealing the field panorama that has been faced, as well as the process of building a matrix for the analysis of texts studied within the indicated period, including the main findings in the course of the ongoing investigation. Regarding the mapping of the studies published in Chile such as books, book chapters and articles in academic journals in the decade studied, this research showed a total corpus of 452 publications. The methodology of analysis involved the creation of a reading matrix in which the texts where categorized. The preliminary design of this matrix was subjected to the discussion of national and international experts and provided rich insights about the coincidences and discordances with the premises, which this project was started. Furthermore, it pointed out the path to narrowing the instrument of analysis that has been applied to date (October 2016). This research projects methodological and practical aspects that contribute to the development and discussion of film studies in Chile and Latin America.

\section{How to cite this text:}

María Marcela Parada Poblete (2017): “The film studies in Chile: an approach to the configuration and thinking of a field”, en Miguel Hernández Communication Journal, n⿳8, pp. 85 to 119. Universidad Miguel Hernández, UMH (Elche-Alicante). Accessed $20 \_$in: [paper link in mhjournal.org] 


\section{Introducción}

El cine chileno ha experimentado una notable emergencia en los últimos años (2005-2015) y el panorama de la industria actual parece ser auspicioso. La sensación generalizada es que en Chile hoy se hacen más películas y los estrenos semanales que aparecen en cartelera, así como la exhibición y competencia en festivales, tanto nacionales como internacionales, parecen confirmarlo. Se procura corroborar esta impresión y revisando los datos de distintos informes del Consejo Nacional de la Cultura y las Artes (CNCA) y la Cámara de Exhibidores Multisalas de Chile A.G. (CAEM), además de autores que han investigado el tema, si bien presentan algunas discrepancias numéricas menores entre todos ellos, el hecho es que ratifican que la industria del cine en Chile manifiesta un crecimiento exponencial en las últimas dos décadas.

Consideremos algunas cifras para ilustrar el panorama. La investigación de Trejo (2009) observa que entre 1940 y 1973, se produjeron y estrenaron en Chile un total aproximado de 95 largometrajes, entre ficciones y documentales. Entre 1973 y 1990 -época atravesada por la dictadura militar en Chile-, se realizaron y exhibieron en Chile un total de 10 largometrajes. En este período, de 1973 a 1983, con el apoyo de actores públicos y privados en el extranjero, el cine chileno en el exilio produjo un total de 56 largometrajes, 34 mediometrajes y 86 cortometrajes; cifras que, como consignan Mouesca y Orellana, "no tienen paralelo en ningún período anterior o posterior de la historia del cine nacional.” (1998: 358).

Entre los años 1990 y 1999 se estrenaron en Chile 28 largometrajes chilenos, de los cuales veintisiete corresponden a ficción y sólo uno a documental. (Trejo, R. 2009: 101). Seguidamente, entre el año 2000 y 2004 se estrenaron en Chile un total de 69 películas nacionales, entre los años 2005 y 2009 los estrenos alcanzaron a 73, y entre el año 2010 y 2015 los estrenos chilenos en el país subieron a 138. (CAEM, 2015). Si bien las distintas investigaciones aluden a producción de cine chileno, estrenos de cine chileno y a películas chilenas exhibidas ya sea de ficción, documental o ambos -lo que, evidentemente, no equivale a la misma medida de cuantificación por período-, la escalada de cifras es elocuente.

En este escenario iniciamos la investigación: "Mapa de los estudios de cine en Chile (2005-2015)", proyecto financiado por el Fondo de Fomento Audiovisual de Chile, convocatoria 2016. Ante el evidente aumento en la realización y estrenos, la interrogación se dirigió al estado del campo de los estudios de cine en Chile en el período 2005-2015, entendiendo los estudios de 
cine como la investigación que tiene como resultado la publicación de tales trabajos.

El estudio partió de la macro hipótesis que cartografiar y analizar tal publicación posibilitaría dar cuenta - en mayor o menor medida, eso estaba por verificarse- de un movimiento de reflexión sobre el estado del arte y la industria, así como del panorama de conformación de una comunidad de pensamiento acerca de pensar no sólo el cine sino -para el caso- el cine chileno, como un objeto de estudio que convoca distintas dimensiones: la de ser una manifestación artística, histórica y social, una institución económica, un producto tecnológico y al mismo tiempo cultural. Y si una de las premisas fue que este crecimiento exponencial en la industria cinematográfica chilena debía de asumir de algún modo un correlato en la teoría y reflexión de campo cultural, la pregunta fue de qué manera lo hacía.

Dar cuenta de esta investigación en curso es una invitación a la discusión y reflexión teórica en el horizonte de conformar una comunidad de pensamiento acerca de pensar los estudios de cine, cuando los códigos heredados y las palabras, así como los intereses particulares de cada cual producto de cada zona de experiencia se contraponen en múltiples visiones de mundo. Como refiere Rojas:

Es cuando se reflexiona a partir del lugar mismo en el que uno se encuentra, cuando las contradicciones, las aporías, las paradojas que se han alojado en la cotidianeidad comienzan a exigir al pensamiento. Esto puede llegar a ser algo germinalmente muy poderoso, pues envía a los individuos no sólo a reflexionar, sino también a escribir y a dialogar con otros individuos que comparten ese mismo pathos reflexivo. Es entonces cuando comienza a producirse una comunidad de pensamiento que trasciende las disciplinas. (2016: 3)

En lo que sigue, este documento se estructura considerando, en primer lugar, los datos de investigaciones precedentes que contribuyeron a delinear el campo de observación y estudio. Seguidamente, se refieren las bases particulares de esta investigación, especificando en ello el objetivo general, corpus de estudio e hipótesis. Se comparte el proceso metodológico llevado a cabo para luego exponer y discutir los principales resultados alcanzados en el estudio y las conclusiones del trabajo realizado. 


\subsection{Contribuciones precedentes}

Los trabajos precedentes de Stange y Salinas (2008 y 2009) y el de Parada (2011) esbozaron algunos lineamientos para el abordaje de esta investigación. A continuación se reseña parte de las identificaciones que contribuyeron a delinear el panorama de campo que enfrenta este estudio.

El trabajo de Stange y Salinas (2008), aborda un panorama general de la literatura sobre cine chileno desde 1957 al 2008, distinguiendo gran parte de la publicación de libros en Chile y en el extranjero, junto a las temáticas predominantes en distintos momentos de esta línea de tiempo. La investigación reconoce que entre 1990 y 1999 "se publicó la misma cantidad de libros sobre cine chileno que en todas las décadas precedentes, duplicando el acervo en la materia y dando la apariencia de que, por primera vez, se estaba construyendo un campo de estudio y crítica." (2). Seguidamente, en el período 2000-2007 se "volvió a duplicar la producción de literatura sobre cine, publicando la misma cantidad de ejemplares que entre los años 1957-1999 en su conjunto." (3). Los autores señalan que la tendencia que cruza el circuito de publicaciones es la historia de distintos acontecimientos y momentos del cine chileno, con una apertura temática hacia el estudio del documental desde el año 2005. El panorama finaliza con un listado de publicaciones en orden cronológico entre los años 1957 y 2008, en el que si bien se incluyen publicaciones en el extranjero, predomina la referencia a libros editados en Chile.

El año 2009, Stange y Salinas publican un artículo que se entiende continúa la investigación anterior y esta interrogación al campo de los estudios de cine en Chile. Reconociendo -junto a Iván Pinto- que el escenario de auge del cine chileno, "parece ser no sólo de orden cuantitativo sino también el inicio de una tendencia hacia la estabilización y diversificación de este campo cultural" (272), los autores abordan el análisis en vistas a identificar las posibles correspondencias entre este desarrollo del cine en Chile y la conformación de un campo de estudios sobre la materia, revisión que extiende sus redes a la institucionalidad, los modelos teóricos y las prácticas críticas.

En dicho artículo se proyecta una segmentación de ocho categorías para identificar la temática de los libros publicados en Chile entre los años 1957 y 1973: 1) Historia, 2) Ensayo temático, 3) Diccionario-manual, 4) Catálogofilmografía, 5) Estudio económico-social, 6) Análisis-crítica, 7) Desconocido, 8) Guión-estudio de film; identificando que en este período el mayor porcentaje de las publicaciones se concentra en Historia con un 34\%. Le 
siguen Ensayo temático con un 14\% y Diccionario-manual con un 13\%. Mientras Análisis-crítica adhiere el 9\%, apenas el 7\% se concentra en Estudio económico-social.

Entre 1950 y 1990, se distingue prevalecen los estudios respecto de historias del cine, y desde 1990 algunas escasas publicaciones evidencian la inclusión particular de una propuesta de reflexión teórica/metodológica. (273).

El año 2005, entra a cuadro el Fondo Audiovisual del Consejo Nacional de la Cultura y las Artes (CNCA); alero bajo el cual, en la línea de investigación, los autores consignan 25 proyectos que reciben el apoyo estatal entre los años 2005 y 2008 (275). De estos 25 proyectos, se observa que para aquellos que se relacionan a los estudios del cine chileno, la investigación histórica concentra nueve trabajos, equivalentes al 36\% del total. Mientras el 12\% corresponde a estudios acerca de la industria audiovisual y el 8\% a estudios focalizados en realizadores. Yendo más allá de la cuantificación, y analizando tanto la bibliografía existente como los proyectos de investigación financiados por el CNCA, los autores señalan:

Más importante aún: los trabajos que presentan coincidencias temáticas no manifiestan marcos epistemológicos definidos, un lugar interpretativo claro o un bagaje teórico y terminológico común. [...] Al no constituir estos estudios un conjunto de modelos o tradiciones que sirvan de sustrato a la investigación subsecuente, pareciera el investigador estar obligado a reconstruir el ámbito y el objeto de su estudio cada vez, a volver a descubrir siempre los datos y las películas, a tratar toda fuente como si fuera de primera mano y todo problema como si se formulara por primera vez. (275-276)

Junto a lo anterior, se advierte que las prácticas críticas y reflexivas "no se organizan en torno a los saberes específicos del cine ni en torno a los modelos teóricos que, como hemos visto, son difusos e inorgánicos" (281), y que tales prácticas recurren a teorías y metodologías provenientes de otros campos, a riesgo de producir malas traducciones de préstamos teóricos para abordar el campo específico del cine.

La interrogación, ciertamente, es una exigencia al pensamiento. $\mathrm{Y}$ si nos concentramos en pensar y examinar los estudios de cine, las preguntas que surgen a continuación son: ¿cuáles serían aquellos saberes que le son propios al cine?, ¿cuál su propiedad?; entendiendo, junto a Zavala, que:

El terreno de los estudios cinematográficos es similar al de los estudios literarios, en el sentido de que el cine no es una disciplina, sino un objeto de 
estudio (que, sin embargo, requiere tener la autonomía de una disciplina). A su vez, los estudios sobre cine se apoyan en varias disciplinas específicas, como la historia, la sociología y muchas otras. (2009: 1)

En esta investigación se ensaya que la respuesta se halla en dirigirse a los estudios del cine, precisamente, con más preguntas: ¿Qué entendemos -O declaramos entender- por pensar el cine? ¿Cuál es el espesor reflexivo que le sería propio al cine para pensar el propio cine desde el interior del campo cultural? ¿Desde dónde sería adecuado -y validado- que tal estudio del cine procediera? ¿Cuáles son los temas y objetos de estudio que sería posible considerar en los estudios de cine? ¿Cuáles serían los modelos teóricos/metodológicos que sería apropiado considerar para validar la investigación del cine en Chile? Preguntas todas que subyacen en nuestro propio proyecto de investigación, en vistas a diseñar una matriz de análisis para los textos en estudio.

El artículo de Parada (2011), aborda una visión panorámica de los estudios sobre cine en Chile entre los años 1960 y 2009, focalizando la investigación en los libros publicados en el país en este período. Reconociendo tres etapas que ha experimentado el cine chileno en el período cartografiado -las décadas del 60,70 y 90-, el estudio reconoce en cada segmento cuestiones que inciden en la realización, lo que se distingue operaría afectando o promoviendo la reflexión de campo y consecuente publicación.

El estado de los estudios sobre cine en el país es sintomático no sólo del desarrollo técnico, académico y profesional, sino también de las diversas políticas gubernamentales que han actuado sobre este campo artístico en el ámbito de la realización. En este sentido, el panorama cinematográfico contemporáneo ha dado como rendimiento la publicación continua -no obstante incipiente- de textos sobre el tema en el país, publicaciones que pueden hallarse en ediciones constantes desde el año 90 en adelante. (13)

Se observa que entre 1954 y 1973 se publicaron sólo siete libros sobre el tema, encabezados por dos publicaciones Yo soy tú (Délano, 1954) y Grandezas y miserias del cine chileno (Santana, 1957), escritas como relatos parcelados y hasta anecdotarios del cine en Chile. Tales publicaciones tienen la particularidad de haber sido abordadas por realizadores cinematográficos, lo que equivale a documentos de primera fuente y la primera iniciativa de relatar cuestiones relacionadas con el panorama de campo del cine en Chile. (14)

Estos dos libros del período comparten el escenario de publicaciones con dos textos de Historia del cine chileno (Godoy, 1966 y Ossa, 1971), uno que aborda 
una compilación de los largometrajes de argumental y documental (Oñate, 1973), un texto sobre el tema del montaje cinematográfico (Sánchez, 1970) y un texto que trabaja sobre el guión del film El chacal de Nabueltoro (Littin, 1970).

Con lo anterior, es posible observar que el relato de experiencias, la historia, el inventario, el montaje y el guión son los temas que inauguran la escena de publicaciones en Chile.

Entre 1974 y 1989, correspondiendo con el período de dictadura militar en Chile, se registran sólo cuatro publicaciones. Entre 1990 y el 1999, el artículo de Parada (2011) consigna que las publicaciones acerca de los estudios del cine chileno comparten el escenario con aquellas que abordan cuestiones relativas al cine en general, como puede observarse en los títulos: Clásicos del cine (Navarro, 1996), Cine y memoria del siglo XX (Mouesca y Orellana, 1998) y el diccionario Qué es el cine (Muñoz, 1999), entre otros.

En cuanto a la investigación del cine chileno en particular, las revisiones de períodos concentran la atención con 6 publicaciones, junto a las que destaca Historia del cine y video en Valdivia (González, 1996), primera iniciativa de estudio respecto de la realización regional.

Entre el año 2000 y 2009, se publican en Chile 56 libros relativos al cine. Distinguiendo de entre los libros cartografiados aquellos que refieren al cine chileno en particular, se observa que, junto a la revisión de períodos, los temas se diversifican y amplían, con materias como la censura, realizadores chilenos y el cine regional (Parada, M. 2011: 16); además de la publicación en el año 2008 de diez libros que corresponden a diez guiones de películas chilenas.

Para el proyecto de investigación que referimos en este artículo, si bien podría argumentarse que la publicación de guiones no corresponde precisamente a un estudio del cine chileno, se considera relevante que se den tales ediciones; por cuanto corresponden, en primer lugar, a una vía de circulación de los filmes y, en segundo lugar, el guión publicado asiste literariamente como material de estudio que permite abrir la reflexión de campo a otras articulaciones.

Para el año 2010, y en proyección respecto del período en estudio 1960-2009, el artículo refiere dos publicaciones que abren el enfoque de estudios y temas de interés: Animación, la magia en movimiento (Barry) y El Cine y el Derecho Penal (Cuneo). (16) 
El panorama finaliza con un listado de los libros publicados en Chile en el período en estudio, entre los cuales, si bien predominan los estudios de cine chileno, también fueron considerados aquellos libros que abordan temáticas relativas al cine en general.

\subsection{Objetivo general de la investigación en curso}

Cartografiar y analizar el campo de la investigación sobre cine en Chile en el período 2005-2015, identificando las inclinaciones de reflexión teórica realizadas en torno al cine chileno que han sido publicadas en el país en la década en estudio. Interesa reconocer los intereses de investigación dominantes y recesivos; presencias, predominancias y ausencias que puedan verificarse en cuanto a períodos en estudio del cine chileno; temas y objetos de estudio que convoca el cine chileno ya sea como realizaciones o como campo; perspectivas teóricas dominantes; tipo de investigación/análisis y fuentes de financiamiento. Esto último en vistas a identificar la participación del Estado y Universidades entre otras fuentes de financiamiento, lo que proporcionaría un indicador respecto de políticas estatales e institucionales de fomento a la investigación cinematográfica chilena.

\subsection{Corpus en estudio}

El corpus de análisis fue acotado a las investigaciones que han sido publicadas sobre el cine chileno en Chile; esto es, textos publicados como libros, capítulos de libro y artículos en revistas académicas, considerando que los dos primeros responden a un criterio de circulación de las obras, en tanto los artículos a un criterio de calidad.

\subsection{Hipótesis}

Como se mencionó en líneas precedentes, esta investigación parte de la macro hipótesis que cartografiar y analizar las investigaciones publicadas sobre cine chileno en Chile en el período 2005-2015 posibilitaría dar cuenta - en mayor o menor medida- de un movimiento de reflexión sobre el estado del arte y la industria, así como del panorama de conformación-quizá, incluso, desfiguración, o conformación incipiente en voluntad de concreción- del campo de estudios de cine en Chile.

La pregunta que comandó nuestra investigación fue de qué manera se ha desarrollado el campo de la investigación sobre cine en Chile en la última década. La hipótesis apunta a que los modelos de investigación asociados a la 
historiografía clásica y orientados en muchos casos a la valoración de directores o a la historia de la cinematografía nacional de modo cronológico y descriptivo, coexisten con nuevas propuestas de estudios cualitativos desde distintas aproximaciones y con focos específicos, y que los estudios sobre la industria, en tanto, son escasos en la academia.

\subsection{Organización del proyecto}

El proyecto total, en desarrollo durante el año 2016 y los primeros seis meses del año 2017 comprende 4 etapas/acciones:

La primera etapa/acción (enero-junio del 2016) corresponde al levantamiento de las publicaciones sobre cine chileno editadas en Chile en el período 20052015. Considerando las investigaciones precedentes sobre el tema, recordemos que éstas consignaban de modo parcial las publicaciones de libros editados en Chile y en el extranjero, y que referían tanto a los estudios del cine chileno como del cine en general. Nuestra pesquisa da como resultado una cartografía inédita de los libros, capítulos de libros y artículos académicos referidos al cine chileno y publicados en el propio país.

La segunda etapa/acción (enero-mayo del 2016) abordada en paralelo al levantamiento de información, corresponde al desarrollo de una matriz de análisis para los textos en estudio, instrumento de análisis diseñado específicamente para esta investigación y consultado con especialistas para su validación.

La tercer etapa/acción (junio del 2016 a febrero del 2017), corresponde al análisis de los textos publicados en el período en estudio. La revisión de los textos hallados y consiguiente vaciado de lectura a la matriz, procura reconocer en las publicaciones cartografiadas las principales temáticas, referencias teóricas, disciplinarias y metodológicas, así como las fuentes de financiamiento; distinciones que despliegan y conciernen a las investigaciones producidas en el país en la última década.

La cuarta etapa/acción (febrero-junio del 2017) es el desarrollo de un sitio web que reunirá los resultados de la investigación en un repositorio digital inédito de publicaciones sobre cine en Chile, accesible tanto a especialistas como a público general. 


\section{Metodología}

\subsection{Cartografía de publicaciones}

La base de datos de publicaciones a analizar consideró la pesquisa de los textos sobre cine chileno publicados en Chile en el período 2005-2015, con sus correspondientes identificaciones editoriales además del financiamiento de publicación referido en la misma publicación.

\subsection{Matriz de análisis y método Delphi}

La metodología de análisis para las publicaciones cartografiadas se aborda a partir de una matriz de lectura y vaciado de textos pensada y proyectada como un instrumento de observación que tuviese el potencial de responder a la diversidad de enfoques que, podía preverse, se encontraría luego en los textos en estudio.

Se asume el problema de sistematizar categorías de observación, en la consciencia de que cualquier clasificación corría el riesgo de ser, al final del día, arbitraria y limitativa, batiéndose a duelo con la realidad de las publicaciones, perspectivas y experiencias de investigación. Se consideró que el diseño de una matriz categorial permitiría acceder a un primer deslinde territorial, que otorgara la posibilidad de distinguir en el corpus de publicaciones los intereses de investigación dominantes y recesivos, presencias, ausencias y desafíos para la consolidación institucional de campo de los estudios de cine en Chile.

Para la validación y acotación de la matriz se utilizó una versión flexible del método Delphi, técnica de consulta anónima realizada a personas acreditadas, cuya formación y ocupación los certifican como agentes clave del proceso.

En la elección de este método se optó, asimismo, por el riesgo: proponer una matriz de análisis y someterla a discusión de un grupo especializado; acción que, se vislumbraba, generaría acuerdos y desacuerdos, según la experiencia particular de cada uno de los expertos y expertas contactados para este proyecto. Se consideró que estos acuerdos y desacuerdos propiciarían una instancia preliminar de diálogo, colaborando a construir una red de pensamiento acerca de pensar los estudios de cine. 


\subsection{Especialistas en consulta y fases de discusión}

En la aplicación del método Delphi, se contactó vía correo electrónico a 56 investigadores expertos y expertas en cine, a quienes se les solicitó colaborar en esta investigación mostrando el acuerdo o desacuerdo con la primera versión de la matriz de análisis y sus correspondientes categorías de estudio. La consulta recibió en una primera fase la respuesta de 22 especialistas de Argentina, Brasil, España, Estados Unidos y México, además de expertos y expertas chilenos residentes tanto en Chile como en el extranjero.

En la práctica, la consulta consideró dos fases de discusión. Para la segunda fase se sumaron 6 especialistas a la discusión. Los comentarios, acuerdos y desacuerdos expresados por todos ellos en ambas consultas, con su correspondiente sistematización, guiaron el rediseño de matriz, el que fue acotado en fases sucesivas tanto en relación a los comentarios expresados por la comunidad de especialistas como en su puesta a prueba por el equipo de investigación en la aplicación del instrumento en una muestra de textos seleccionados para tal efecto.

\subsection{Sistematización de comentarios de la comunidad de especialistas}

Para ambas fases de consulta respecto de la matriz, se realizó el procesamiento de las opiniones expresadas por los y las especialistas, agrupándolas según el acuerdo o desacuerdo que manifestaban con cada sección del diseño de instrumento de análisis propuesto. Procesada la información, se elaboró en cada fase de consulta un informe de sistematización que fue enviado a la comunidad de especialistas participantes.

\subsection{Rediseño de matriz y puesta a prueba}

Se procedió al rediseño del instrumento de análisis, procurando que la nueva versión recogiese las observaciones de los y las especialistas, realizando un trabajo de agrupación y selección de comentarios en vías de que la aplicación de la matriz de análisis pudiese ser operativa y el inicio de nuevos abordajes para los estudios de cine.

Se realizó la lectura y vaciado de análisis a la matriz versión 2.0 de cuarenta y seis textos correspondientes a los capítulos referidos a cine chileno en los 
libros publicados en el marco del Encuentro de Investigación sobre Cine que realiza anualmente la Cineteca Nacional de Chile desde el año 2011.

\subsection{Discusión de avances del proyecto}

La matriz de análisis y los resultados parciales obtenidos según su aplicación en la muestra de textos en estudio fueron expuestos y compartidos con la comunidad académica como avances del proyecto en dos instancias:

a) VI Encuentro de Investigación sobre cine chileno y latinoamericano, organizado por la Cineteca Nacional de Chile. Santiago de Chile. Participación en Encuentro: 26 de abril de 2016 Ponencia presentada: Construcción de un mapa de estudios de cine en Chile (2005-2015). Foco de la ponencia: exposición de la metodología del proyecto en curso y estado de avance de la investigación. Expositores: Marcela Parada y Pablo Andrada (investigadores)

b) 50 Congreso LASA 2016 (Latin American Studies Association), Nueva York, Estados Unidos. Participación en Congreso: 27 de mayo del 2016 Ponencia presentada: Mapa de los estudios sobre cine chileno y latinoamericano en Chile: perspectivas y reflexiones del ámbito académico.

Foco de la ponencia: resultados cuantitativos y cualitativos del vaciado de los 46 textos iniciales en la matriz. Expositora: Mónica Villarroel (asesora del proyecto)

En ambas conferencias, se recogieron los comentarios de los asistentes respecto del método de análisis y su propiedad, contrarrestando y balanceando tales opiniones con los objetivos del proyecto en curso.

\subsection{Diseño de matriz versión 3.0}

Se realizó una siguiente acotación en el diseño de la matriz, agregando categorías de análisis y agrupando otras, siempre en vías de que la aplicación del instrumento de análisis pudiese ser operativa reuniendo las sugerencias de la comunidad de especialistas.

Seguidamente, se reitera la puesta a prueba del instrumento de análisis en su versión 3.0 con los cuarenta y seis textos trabajados en la etapa anterior, 
verificando que el equipo de investigación compartiera el mismo criterio de lectura, vaciado y análisis de los textos en estudio.

\subsection{Instrumento de análisis en aplicación}

A la fecha -octubre del 2016- se desarrolla la tercera etapa/acción del proyecto, correspondiente a la lectura y análisis del universo de textos publicados en el período en estudio.

\section{Resultados}

Los resultados de análisis que se presentan en este artículo corresponden al universo de libros y capítulos de libros publicados sobre cine chileno en Chile en el período 2005-2015. Dado que se trata de una investigación en curso, estos resultados comparten una muestra parcial del panorama de campo de los estudios sobre cine en Chile y por lo pronto son, principalmente, de orden cuantitativo.

\subsection{Cartografía total y observaciones}

El total de publicaciones cartografiadas editadas en Chile entre los años 20052015 arroja la cantidad de 452 textos, a los que se suman eventualmente 5 que habiendo sido referidos en algún texto o noticia de página web, no pudieron ser hallados para esta investigación y están, por lo pronto, extraviados.

Figura 1. Desglose de las 457 publicaciones cartografiadas

\begin{tabular}{|l|r|}
\hline Total de libros cartografiados & 127 \\
\hline $\begin{array}{l}\text { Libros exclusivos: ediciones cuya totalidad } \\
\text { aborda el tema en estudio }\end{array}$ & 74 \\
\hline Libros cartografiados que no aplican al análisis & 20 \\
\hline $\begin{array}{l}\text { Total de Libros/capítulos: ediciones con uno o } \\
\text { más capítulos referentes al tema en estudio }\end{array}$ & 33 \\
\hline Total de capítulos de libros cartografiados & $\mathbf{2 1 7}$ \\
\hline $\begin{array}{l}\text { Capítulos de libros cartografiados que no } \\
\text { aplican al análisis }\end{array}$ & 10 \\
\hline Total de artículos cartografiados & $\mathbf{1 0 8}$ \\
\hline Publicaciones extraviadas & $\mathbf{5}$ \\
\hline
\end{tabular}




\subsection{Concentración de publicaciones}

Figura 2. Concentración de libros publicados

\begin{tabular}{|l|l|c|}
\hline Actividad de publicación & Año & Libros publicados \\
\hline Mayor actividad & 2008 & 18 \\
\hline Segunda mayoría en actividad & 2013 & 11 \\
\hline Tercera mayoría en actividad & 2011 & 10 \\
\hline Año de más baja publicación & 2005 & 3 \\
\hline
\end{tabular}

Figura 3. Concentración de Libros/capítulos publicados

\begin{tabular}{|l|l|c|}
\hline Actividad de publicación & Año & Libros/ capítulos publicados \\
\hline Mayor actividad & 2013 & 7 \\
\hline Segunda mayoría en actividad & 2011 & 5 \\
\hline Tercera mayoría en actividad & 2012 & 5 \\
\hline Menor actividad & 2006 & 1 \\
\hline Menor actividad & 2008 & 1 \\
\hline Año sin publicación & 2005 & 0 \\
\hline
\end{tabular}

Figura 4. Concentración de capítulos publicados

\begin{tabular}{|l|l|c|}
\hline Actividad de publicación & Año & Capítulos publicados \\
\hline Mayor actividad & 2011 & 50 \\
\hline Segunda mayoría en actividad & 2013 & 40 \\
\hline Tercera mayoría en actividad & 2014 & 34 \\
\hline Menor actividad & 2005 & 1 \\
\hline Menor actividad & 2006 & 1 \\
\hline Año sin publicación & 2008 & 0 \\
\hline
\end{tabular}


Figura 5. Concentración de artículos publicados

\begin{tabular}{|l|c|c|}
\hline Actividad de publicación & Año & Artículos publicados \\
\hline Mayor actividad & 2010 & 19 \\
\hline Segunda mayoría en actividad & 2014 & 18 \\
\hline Tercera mayoría en actividad & 2012 & 17 \\
\hline Menor actividad & 2005 & 4 \\
\hline Menor actividad & 2008 & 4 \\
\hline Menor actividad & 2009 & 4 \\
\hline Año de más baja publicación & 2006 & 2 \\
\hline Año de más baja publicación & 2007 & 2 \\
\hline
\end{tabular}

\subsection{Focalización de los estudios}

Se reseñan a continuación los principales resultados hallados en el análisis de libros y capítulos de libros, identificando intereses de investigación dominantes y recesivos.

\subsubsection{Período del cine chileno}

El período del cine chileno con mayor concentración de estudio corresponde en la matriz de análisis a la sub categoría Contemporáneo, reuniendo el 42\% de publicaciones. La sub categoría de Diversos concentra un 29\% de publicaciones, en el cual el 77\% incluye el período Contemporáneo en una lectura relacional con otros períodos. El período Clásico-Industrial, en tanto, corresponde al menos estudiado recogiendo el $2 \%$ de las publicaciones.

\subsubsection{Género cinematográfico}

El Género cinematográfico con mayor concentración de estudio corresponde en la matriz de análisis a la sub categoría Ficción, reuniendo el 41\% de las publicaciones. Le siguen las sub categorías de Documental con un 19\% y Mixto con un 15\%. La sub categoría de Animación, en tanto, corresponde a la menos estudiada con un $1 \%$ de las publicaciones.

\subsubsection{Temas y objetos de estudio}

El tema y objeto de estudio que concentra el mayor porcentaje de publicaciones corresponde en la matriz de análisis a la sub categoría de 
Realizadores, reuniendo el 30\% de los estudios. La sub categoría de Identidad concentra el $24 \%$ de los estudios e Industria el 20\%. Las sub categorías de menor abordaje en los estudios son las de Estudios comparados (2\%) y Cine de regiones de Chile (1\%).

\subsection{Tipo de investigación/análisis}

En el análisis de libros y capítulos de libros se observa que el tipo de investigación cualitativa es el que predomina en los estudios, concentrando un $80 \%$ del corpus examinado.

\subsection{Financiamiento de las publicaciones}

Para detectar las fuentes de financiamiento de libros y capítulos de libros, se pesquisa aquellas que son señaladas expresamente en los textos en estudio, las que se encuentran en el 48\% de las publicaciones. De ello, se observa que la sub categoría Estado concentra el mayor apoyo para las publicaciones sobre cine chileno con un 64\%. La sub categoría Mixto, que considera Privado + Universidad y Estado + Universidad, concentra un 21\%, y el financiamiento en la categoría Universidad presenta un escaso 9,6\%.

\section{Discusión}

En las líneas que siguen se expone el proceso que siguió la construcción de la matriz de análisis y a continuación se desarrollan los resultados -a la fecha parciales: octubre del 2016- de su aplicación.

\subsection{Instrumento de análisis: primer diseño}

La primera versión de matriz de análisis propuesta por el equipo de investigación distinguió cuatro campos de observación para los textos en estudio: Tema y Objeto de Estudio, Modelo Teórico, Metodología y Fuente de Financiamiento; considerando que en estas cuatro zonas, con su consecuente despliegue de categorías y sub categorías, es posible distinguir cuestiones significativas para configurar una primera lectura del estado de campo de los estudios de cine en Chile. 
Figura 6. Primer diseño matriz de análisis

\begin{tabular}{|c|c|c|}
\hline \multicolumn{3}{|c|}{ ZONA 1: TEMAY OBJETO DE ESTUDIC } \\
\hline \multirow[t]{9}{*}{ 1.A } & \multicolumn{2}{|c|}{ Historiografía } \\
\hline & 1.A1 & Silente (1896-1933) \\
\hline & 1.A2 & Clásico (1933 a 1954) \\
\hline & 1.A3 & Moderno (1955 a 1969) \\
\hline & 1.A4 & Militante (1970-1973) \\
\hline & 1.A5 & Dictadura y exilio (1973-1990) \\
\hline & $1 . \mathrm{A} 6$ & Post Dictadura (1990-1998) \\
\hline & 1.A7 & Siglo XXI (1999-2015) \\
\hline & 1.A8 & Mixto \\
\hline $1 . \mathrm{B}$ & \multicolumn{2}{|c|}{ Realizadores } \\
\hline \multirow[t]{16}{*}{ 1.C } & \multicolumn{2}{|c|}{ Cine de regiones de Chile } \\
\hline & 1.C1 & Región I \\
\hline & 1.C2 & Región II \\
\hline & 1.C3 & Región III \\
\hline & 1.C4 & Región IV \\
\hline & 1.C5 & Región V \\
\hline & 1.C6 & Región VI \\
\hline & 1.C7 & Región VII \\
\hline & 1.C8 & Región VIII \\
\hline & \begin{tabular}{|l|l|}
$1 . C 9$ \\
\end{tabular} & Región IX \\
\hline & 1.C10 & Región X \\
\hline & 1.C11 & Región XI \\
\hline & 1.C12 & Región XII \\
\hline & 1.C14 & Región XIV \\
\hline & 1.C15 & Región XV \\
\hline & 1.C16 & Mixto \\
\hline \multirow[t]{5}{*}{ 1.D } & \multicolumn{2}{|c|}{ Documental } \\
\hline & 1.D1 & Directo \\
\hline & 1.D2 & Recreaciones \\
\hline & 1.D3 & Autodocumental, Autobiografías \\
\hline & 1.D4 & Mixto \\
\hline \multirow[t]{3}{*}{ 1.E } & \multicolumn{2}{|c|}{ Guiones } \\
\hline & 1.E1 & Guión \\
\hline & 1.E2 & Análisis de guión \\
\hline \multirow[t]{3}{*}{ 1.F } & \multicolumn{2}{|c|}{ Análisis Cinematográfico } \\
\hline & 1.F1 & Estética \\
\hline & $1 . \mathrm{F} 2$ & Lenguaje \\
\hline $1 . G$ & \multicolumn{2}{|c|}{ Industria } \\
\hline $1 . \mathrm{H}$ & \multicolumn{2}{|c|}{ Identidad } \\
\hline 1.I & \multicolumn{2}{|c|}{ Patrimonio } \\
\hline 1.J & \multicolumn{2}{|c|}{ Intertextualidad } \\
\hline $1 . \mathrm{K}$ & \multicolumn{2}{|c|}{ Recepción } \\
\hline 1.L & \multicolumn{2}{|c|}{ Otros } \\
\hline
\end{tabular}

\begin{tabular}{|l|l|}
\hline \multicolumn{2}{|l|}{ ZONA 2 MODELO TEÓRICO } \\
\hline 2.A & Formalismo \\
\hline 2.B & Semiótica \\
\hline 2.C & Psicoanálisis \\
\hline 2.D & Feminismo \\
\hline 2.E & Cognitivismo \\
\hline 2.F & Teoría Queer \\
\hline 2.G & Teoría Postcolonial \\
\hline
\end{tabular}

\section{ZONA 3: METODOLOGÍ A}

\begin{tabular}{|c|c|c|}
\hline 3.A & Prod & ción de datos \\
\hline & 3.A1 & Etnografía \\
\hline & 3.A2 & Entrevistas \\
\hline & 3.A3 & Focus group \\
\hline & 3.A4 & Encuesta \\
\hline & 3.A5 & Fuentes secundarias \\
\hline & $3 . \mathrm{A} 6$ & Otro \\
\hline & $3 . \mathrm{A7}$ & Sin información \\
\hline $3 . \mathrm{B}$ & Análi & de datos \\
\hline & $3 . \mathrm{B} 1$ & Análisis de texto cinematográfico \\
\hline & $3 . \mathrm{B} 2$ & Análisis cuantitativo \\
\hline & 3.B3 & Análisis cualitativo \\
\hline & 3.B4 & Análisis mixto \\
\hline & $3 . \mathrm{B} 5$ & Otro \\
\hline & $3 . \mathrm{B} 6$ & Sin información \\
\hline
\end{tabular}

\begin{tabular}{|l|l|}
\hline \multicolumn{2}{|l|}{ ZONA 4: FUENTE DE FINANCIAMIENTO } \\
\hline 4.A & Sin financiamiento \\
\hline 4.B & Privado \\
\hline 4.C & Público \\
\hline 4.D & Universidad \\
\hline 4.E & Mixto \\
\hline 4.F & Otro \\
\hline 4.G & Sin información \\
\hline
\end{tabular}


El pronunciamiento de la comunidad de expertos en la primera consulta presentó frecuencias distintas para cada ítem. Se refiere en este artículo una síntesis de los comentarios con aquellas observaciones que exponen las principales polémicas surgidas de la discusión.

\subsubsection{Síntesis de comentarios para: Tema y Objeto de Estudio}

En la categoría de Historiografía, las sub categorías más comentadas corresponden a Militante, y Dictadura y exilio. En el caso de Militante, se discute la división y designación de Militante para este período, entendiendo que el cine militante se cruza con el período que en la matriz se ha denominado Moderno. En el caso de Dictadura y exilio, se sugiere separar esta sub categoría, considerando el cine realizado en Chile durante la dictadura y el cine realizado en el exilio por separado, ya que ambos abarcan el mismo período, pero obedecen a lógicas diferentes.

En la zona general, las categorías más comentadas corresponden a Documental y Análisis cinematográfico. En nivel de participación, le siguen las categorías de Industria y Patrimonio.

En el caso de Documental y respecto de las sub categorías, se propone establecer una sola codificación, donde el Documental incorpora las distintas formas de expresión que pueden alternarse y cohabitar en una obra. Se debate acerca de incorporar las categorías de Ficción, Experimental, Ensayo y Animación, y se discute sobre si Documental podría ser parte de una categoría diferente que sería Géneros Cinematográficos.

En el caso de Análisis cinematográfico, los comentarios se centran en la división de sub categorías que presenta la matriz, discutiendo la pertinencia o incoveniencia de dividir Estética y Lenguaje. Se sugiere incorporar una sub categoría de Análisis comparados de cinematografías chilenas y otras.

En el caso de Industria, se sugiere dividir Industria y Público/audiencias, ya que se percibe apuntan a distintos momentos, entendiendo que Industria ocurre antes que las audiencias. Industria, a su vez, se percibe relacionado con fondos públicos, acciones del Estado y legislación, distribución nacional e internacional, exportación y circulación de cine chileno en el exterior. 
En el caso de Patrimonio, se discute qué se entiende por este concepto y el deslinde que tiene con el concepto de Industria.

\subsubsection{Síntesis de comentarios para: Modelo Teórico}

En esta zona, el debate se centra en las siete categorías referidas en la matriz como Modelos Teóricos. Se cuestiona la propiedad de determinar este conjunto en particular, descartando las más de 50 teorías del cine. Se observa que faltan áreas que no quedan cubiertas en los siete modelos explicitados, como las de Sociología, Antropología, Teoría literaria, Intermedialidad o Historiografía.

\subsubsection{Síntesis de comentarios para: Metodología}

En esta zona, se cuestiona que Producción de datos sea una sublínea de metodología válida para los estudios de cine. Además, se señala que los distintos Análisis propuestos en la matriz corresponden a Categorías Metodológicas.

\subsubsection{Síntesis de comentarios para: Fuente de Financiamiento}

En esta línea, se señala que no todas las categorías son excluyentes, como por ejemplo: el público con el universitario.

\subsection{Instrumento de análisis: iteración y rediseño}

Con los resultados de la segunda vuelta de consulta se procede al rediseño del instrumento de análisis.

Se adopta la división de la sección 1, distinguiendo Período cine chileno (1.A) de Tema y Objeto de Estudio (1.B), entendiendo que ello posibilita reconocer en los textos analizados, en primera instancia, si existe una etapa del cine chileno que convoque mayor o menor análisis, en qué período se concentran de concentrarse- los estudios, y si hay un interés particular de estudio que pueda distinguirse en diversos momentos de la década 2005-2015.

La categoría 1.B Tema y Objeto de Estudio, se dirige en esta segunda versión de matriz particularmente a la materia en estudio de tal o cual período del cine en análisis que se identifique en primera instancia en el texto a examinar. 
En esta segunda versión, las sub categorías correspondientes a Período cine chileno y Tema y Objeto de estudio son, a su vez, repensadas y reorganizadas.

Figura 7. Segundo diseño matriz de análisis. División Zona 1 para el análisis

\begin{tabular}{|l|l|}
\hline \multicolumn{2}{|l|}{ ZONA 1A: PERI ODO CINE CHILENO } \\
\hline 1.A1 & Silente \\
\hline 1.A 2 & Clásico-Industrial \\
\hline 1.A 3 & Moderno \\
\hline 1.A4 & Dictadura \\
\hline 1.A5 & Exilio \\
\hline 1.A6 & Post Dictadura \\
\hline 1.A7 & Contemporáneo \\
\hline 1.A8 & Diversos \\
\hline
\end{tabular}

\begin{tabular}{|l|l|}
\hline \multicolumn{2}{|l|}{ ZONA IB: TEMA Y OBJETO DE ESTUDIO } \\
\hline 1.B1 & Realizadores \\
\hline 1.B2 & Cine de regiones de Chile \\
\hline 1.B3 & Géneros cinematográficos \\
\hline 1.B4 & Guiones \\
\hline 1.B5 & Industria y Estudios de Audiencia \\
\hline 1.B6 & Identidad \\
\hline 1.B7 & Intermedialidad/Intertextualidad \\
\hline 1.B8 & Estudios comparados \\
\hline 1.B9 & Otros \\
\hline
\end{tabular}

La sub categoría Cine de regiones de Chile, elimina la subdivisión de regiones que aparecía en la primera versión de matriz, considerando que lo que interesa aquí es identificar la descentralización, si existe el estudio de la cinematografía de regiones del país, distintas a la región Metropolitana.

La sub categoría Documental pasa a integrar la de Géneros cinematográficos, entendiendo Géneros cinematográficos como la perspectiva del carácter de representación y expresión, junto al contrato de lectura que se establece entre el público y la obra. La nueva categoría de Géneros cinematográficos considera una subdivisión que contiene: Ficción, Documental, Experimental y Ensayo, Animación e Hibridación.

La sub categoría de Análisis cinematográfico se elimina en la nueva versión de matriz, desplazando lo que era considerado en ésta a la categoría de Metodología, entendiendo que el análisis cinematográfico constituye, precisamente, un método de análisis y reflexión de los textos en estudio.

La subcategoría de Industria es repensada y reformulada, conteniendo en la segunda versión de matriz a Industria y Estudios de audiencia.

La subcategoría de Identidad se re estructura en dos divisiones: Identidad y sujeto, e Identidad y memoria.

La sub categoría de Patrimonio se elimina, tomando en consideración los comentarios de los y las especialistas que hacían notar que este término se 
asocia principalmente al acervo cinematográfico, siendo la intención inicial de la matriz relacionarlo con lugares de exhibición cinematográfica en Chile e instituciones relacionadas con la realización y/o exhibición de cine en Chile. En la matriz reformulada, estos temas son incluidos en la categoría Otros.

La sub categoría de Intertextualidad pasa a llamarse Intertextualidad/Intermedialidad, reconociendo que esta nueva nominación permite una mirada más amplia referida al diálogo entre obras fílmicas y entre el cine y otras artes.

La sub categoría de Recepción se elimina y se traslada a la de Estudios de audiencia. Se agrega, en esta segunda versión de matriz, la subcategoría de Estudios comparados. Se mantiene la sub categoría de Otros, considerando para ésta los textos que aborden temáticas sobre el cine chileno que no han sido mencionadas en las categorías anteriores.

La zona de Modelo Teórico, se modifica por Perspectiva Teórica, la que a su vez conlleva tres sub divisiones: Perspectiva (Comparada, Panorámica y Focalizada), Aproximación disciplinaria (Estética, Ciencias Sociales, Historia, Literatura y Otra) y Marco Teórico. Marco Teórico se concibe como una pregunta abierta, en la que se busca identificar autores predominantes, corrientes y/o escuelas teóricas referidas en los textos que se examinan.

La zona de Metodología se reformula en su totalidad, eliminando la subdivisión de Producción de datos y concentrándose en la metodología que puede distinguirse en el análisis de los textos en estudio (Análisis cinematográfico, Análisis Cuantitativo, Análisis Cualitativo, Otros, Mixto y No se especifica).

La zona de Fuente de Financiamiento se re estructura, permitiendo diferenciar la especificidad a que refiere para el análisis el concepto de financiamiento público asociado a fondos provenientes directamente de organismos del Estado (Sin financiamiento, Privado, Estado, Universidad, Mixto, Otro y Sin información).

\subsection{Instrumento de análisis: versión 3.0 en aplicación}

En el proceso de iteración y testeo de aplicación del instrumento de análisis se realizan nuevas acotaciones al diseño de matriz. Se determinan siete campos de observación para los textos en estudio: Período cine chileno, Género 
cinematográfico, Tema y objeto de estudio, Perspectiva teórica, Tipo de investigación/análisis, Fuente de financiamiento y Datos autor/filiación.

En el diseño final de matriz se extrae de la sección Tema y Objeto de Estudio, la sección de Géneros cinematográficos, considerándola como una identificación independiente, entendiendo que ello permite reconocer en los textos analizados qué tipo de realización cinematográfica convoca mayor o menor interés de análisis.

Tema y objeto de estudio, se concentra en la versión final de matriz particularmente en la materia en estudio de tal o cual período de cine chileno y Género cinematográfico identificado en el texto que se examina.

Otras modificaciones adoptadas en esta zona corresponden a la sub categoría de Identidad y memoria, la que pasa a denominarse Identidad cultural, abriendo la designación al estudio del cine chileno e identidad nacional. Se agrega la sub categoría de Patrimonio audiovisual, en vías de identificar en los textos en análisis cuestiones relativas al estudio de instituciones dedicadas al fomento del cine chileno y la realización cinematográfica, resguardo del patrimonio fílmico, archivos, entre otros temas relacionados.

En la zona de Perspectiva teórica y Aproximación disciplinaria, se agrega la sub categoría de Economía de la cultura, para identificar aquellos estudios que analizan el cine chileno considerando cuestiones económicas y gestión cultural. Para la sub categoría de Referencial teórico se determina un máximo de 4 autores y/o corrientes teóricas identificadas en los textos en análisis.

En la zona de Tipo de investigación/análisis, se acotan a tres las sub categorías: Análisis Cualitativo, Análisis Cuantitativo y Mixto considerando, junto a la operatividad de aplicación de la matriz, que estas tres direcciones ya permiten identificar y distinguir el tipo de acercamiento/reflexión de los estudios de cine.

Se incorpora la zona de Datos autor/a/es de la publicación, de modo de registrar los datos que se hallen en el propio texto respecto de desde dónde se escribe y filiación. 


\subsection{Discusión de resultados: libros y capítulos de libros}

Los datos de la Figura 8 muestran las publicaciones cartografiadas por año con edición en Chile en la década en investigación, arrojando un total de 452 estudios (se omiten las 5 publicaciones extraviadas).

Figura 8. Publicaciones por año

\begin{tabular}{ccccc}
\hline Año & Libros & Libro/ capítulos & Capítulos & Artículos \\
\hline $\mathbf{2 0 0 5}$ & 3 & 0 & 1 & 4 \\
$\mathbf{2 0 0 6}$ & 5 & 1 & 1 & 2 \\
$\mathbf{2 0 0 7}$ & 7 & 2 & 18 & 2 \\
$\mathbf{2 0 0 8}$ & 18 & 1 & 0 & 4 \\
$\mathbf{2 0 0 9}$ & 8 & 3 & 7 & 4 \\
$\mathbf{2 0 1 0}$ & 8 & 2 & 24 & 19 \\
$\mathbf{2 0 1 1}$ & 10 & 5 & 50 & 13 \\
$\mathbf{2 0 1 2}$ & 8 & 5 & 20 & 17 \\
$\mathbf{2 0 1 3}$ & 11 & 7 & 40 & 14 \\
$\mathbf{2 0 1 4}$ & 9 & 4 & 34 & 18 \\
$\mathbf{2 0 1 5}$ & 7 & 3 & 22 & 11 \\
Total & 94 & 33 & 217 & 108 \\
\hline & & & & \\
\hline
\end{tabular}

En este escenario, en una primera revisión del corpus, se verificó que no todos los libros y capítulos de libros aplican a la matriz de análisis, dado que las temáticas de algunas publicaciones se enfocan hacia teorías del cine, la obra de realizadores extranjeros en el extranjero, o al análisis de cine internacional y/o latinoamericano sin referir el cine chileno. Bajo esta distinción, el vaciado a la matriz de análisis considera un total de 74 libros y 207 capítulos de libros, lo que corresponde a un total de 281 textos examinados para este proyecto en construcción. 
Figura 9. Frecuencia de publicaciones por año

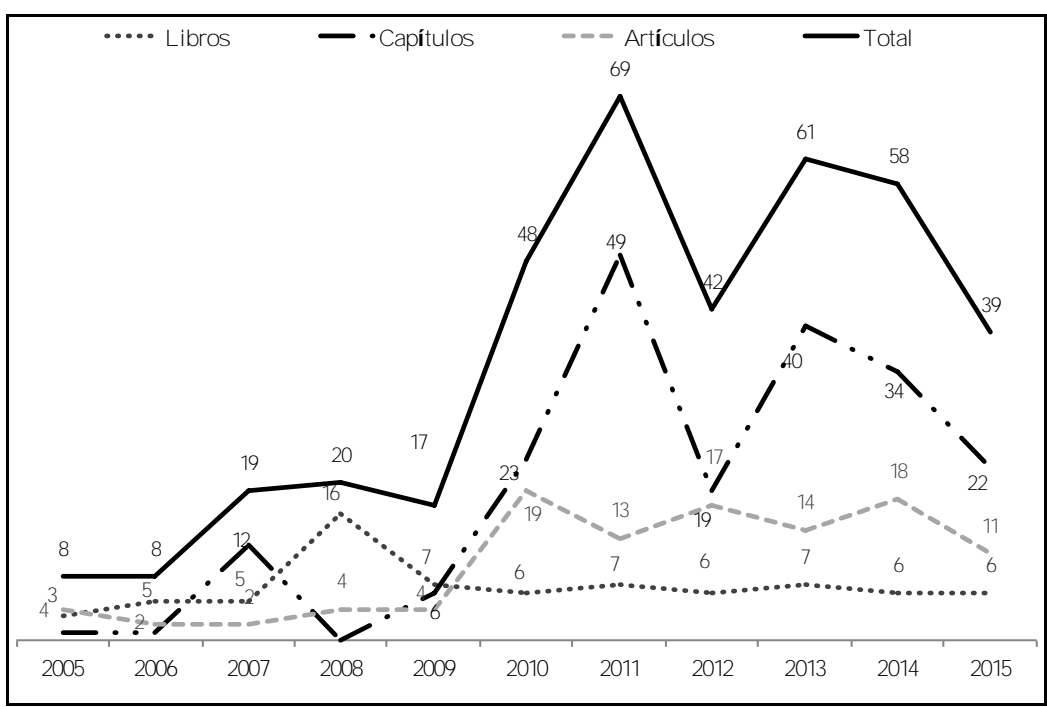

El gráfico de la Figura 9 demuestra el comportamiento de las publicaciones desde el año 2005 hasta el año 2015. En esta visualización se han restado las publicaciones de libros y capítulos de libros que no aplicaban para el análisis de este proyecto.

Respecto de los libros, la mayor actividad se visualiza en el año 2008 con 16 publicaciones. De éstas, 10 de ellas corresponden a una colección de guiones de películas chilenas, iniciativa de la Escuela de cine de la Universidad Mayor y la Cineteca Nacional de Chile (Ocho Libros editores), y una publicación cartografiada en este mismo año corresponde a Retrospectiva afiche del cine chileno, edición que registra una cronología de afiches de películas, iniciativa de publicación de la Facultad de Diseño y la Escuela de Cine de la Universidad del Desarrollo. Los estudios de cine chileno, propiamente tal, publicados como libros en el año 2008 quedan en 5, manteniendo un promedio similar en la década en estudio.

Se distingue el crecimiento que se da en el panorama de campo de los estudios sobre cine chileno desde el año 2010 en adelante, ascenso que se sostiene por la publicación de investigaciones como capítulos de libros y artículos académicos.

El año 2010, presenta la publicación de 23 capítulos, 22 de los cuales están consignados en el libro El cine de Raúl Ruiz: fantasmas, simulacros y artificios 
(Valeria de los Ríos e Iván Pinto, editores). Los 19 artículos publicados en este año se concentran mayormente en las publicaciones de la Revista Aisthesis (Facultad de Filosofía, Instituto de Estética, Pontificia Universidad Católica de Chile), que dedica dos números en este año como Dossier centrados en cine: Número 47, julio 2010: Cine y política en Chile y Número 48, diciembre 2010: Cine y politica en Latinoamérica.

El año 2011 registra la mayor actividad con un total de 69 publicaciones, seguido del año 2013 con 61 y el año 2014 con 58 .

En el año 2011, 37 capítulos de los 49 cartografiados se concentran en la publicación de dos libros: El novísimo cine chileno (Ascanio Cavallo y Gonzalo Maza, editores) con 21 capítulos y El cine que fue: 100 años de cine chileno (Claudia Barril R. y José M. Santa Cruz G., editores) con 16 capítulos. De los 13 artículos registrados, 7 se concentran en publicaciones de la revista Comunicación y Medios (Instituto de la Comunicación e Imagen, Universidad de Chile).

En el año 2013, los capítulos publicados se concentran principalmente en dos libros: Enfoques al cine chileno en dos siglos (Mónica Villarroel, coordinadora) con 20 capítulos y La butaca de los comunes. La crítica de cine y los imaginarios de modernización en Chile (Hans Stange Marcus y Claudio Salinas Muñoz, editores) con 8 capítulos. Los artículos, en tanto, se publican en distintas revistas académicas: Comunicación y Medios, Cuadernos.Info (Facultad de Comunicaciones, Pontificia Universidad Católica de Chile), Atenea (Universidad de Concepción), Aisthesis, Literatura y lingüística (Departamento de Humanidades, Universidad Católica Silva Henríquez), Faro (Facultad de Ciencias Sociales, Universidad de Playa Ancha

Valparaíso) y El Resplandor (Escuela de cine de la Facultad de Arquitectura, Universidad de Valparaíso).

En el año 2014, los capítulos publicados se concentran mayoritariamente en dos libros: Travesías por el cine chileno y latinoamericano (Mónica Villarroel, coordinadora) con 16 capítulos y Audiovisual y política en Chile (Claudia Barril, Pablo Corro y José Miguel Santa Cruz, editores) con 14 capítulos. En cuanto a los artículos, 11 de los 18 registrados se concentran en publicaciones de la revista Comunicación y Medios. 


\subsubsection{Revisión: Período cine chileno}

De acuerdo a las categorías y sub categorías de la matriz, el período más estudiado corresponde al Contemporáneo con un $42 \%$, seguido por Diversos que concentra un $29 \%$, porcentaje en el cual se incluye a su vez el período Contemporáneo en un $77 \%$ de los casos, que es abordado junto a otros períodos en estudio. Las categorías Moderno (11\%), Dictadura (5\%), Exilio $(4 \%)$ y Post Dictadura $(3 \%)$ alcanzan un bajo porcentaje, lo que se explica porque las publicaciones abarcan más de un período, tendiendo una mirada de estudio más panorámica hacia el cine chileno. El período Silente concentra un $4 \%$ de las publicaciones, sin embargo, en la categoría Diversos, hay un 21\% que abarca los comienzos del cine chileno junto a otros períodos. El período Clásico-Industrial (2\%) corresponde al menos estudiado.

Figura 10. Distribución de períodos del cine chileno estudiados

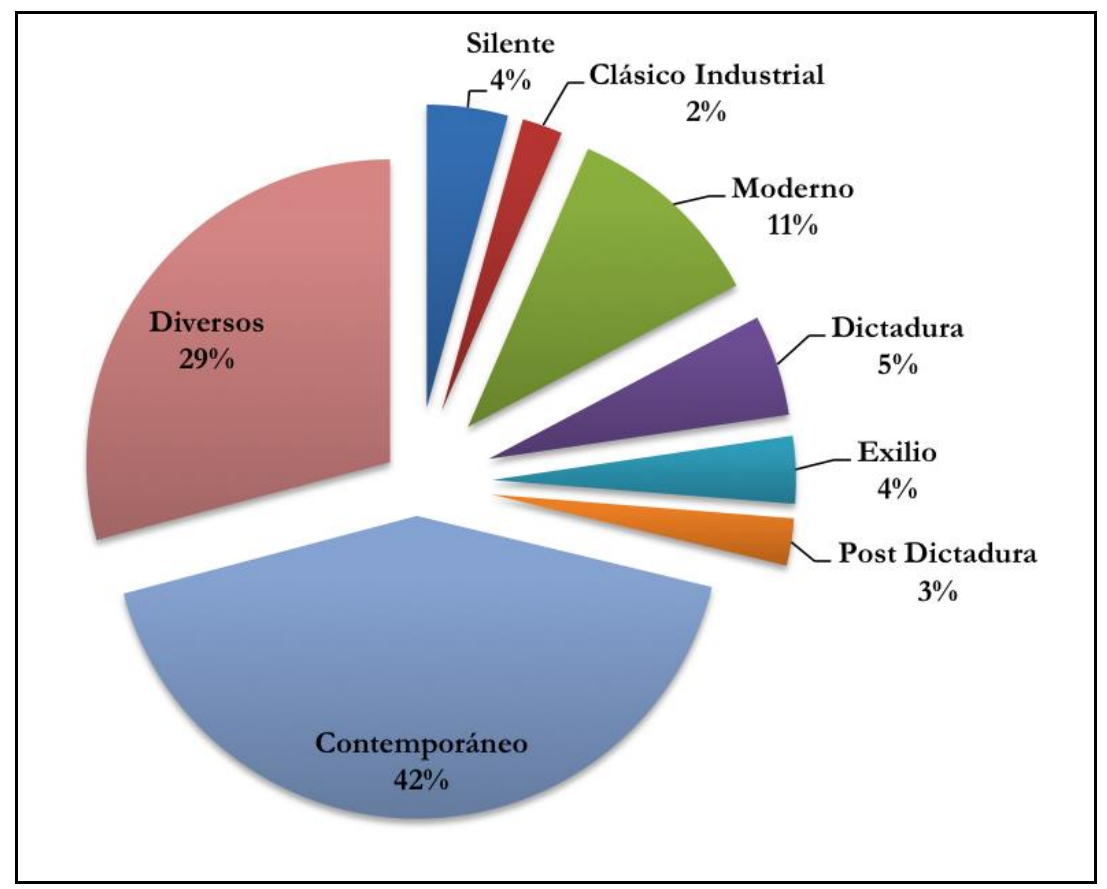

\subsubsection{Revisión: Género cinematográfico}


El género más estudiado corresponde a Ficción con un 41\%, seguido de Documental (19\%), Mixto (15\%), Experimental y Ensayo (5\%), y Animación $(1 \%)$.

La sub categoría de Ficción se observa con más estudios en los años 2010 y 2011, a propósito de los libros El novísimo cine cbileno, El cine que fue: 100 años de cine chileno y El cine de Raúl Ruiz: fantasmas, simulacros y artificios.

El género Documental se concentra en los años 2013, 2014 y 2015 con los libros Audiovisual y política en Chile, El documental politico en Argentina, Chile y Uruguay: de los años cincuenta a la década del dos mil (Antonio Traverso, Tomás Crowder-Taraborreli, editores) y Enfoques al cine chileno en dos siglos y Travesías por el cine cbileno y latinoamericano, ambas ediciones coordinadas por Mónica Villarroel corresponden a los Encuentros de investigación de cine chileno y latinoamericano realizados anualmente por la Cineteca Nacional de Chile.

La categoría Mixta, en tanto, se concentra en el año 2013 también con capítulos en el libro Enfoques al cine cbileno en dos siglos. El género Experimental y Ensayo se concentra en el año 2011, con capítulos en el libro El novísimo cine cbileno.

Figura 11. Distribución de géneros cinematográficos estudiados

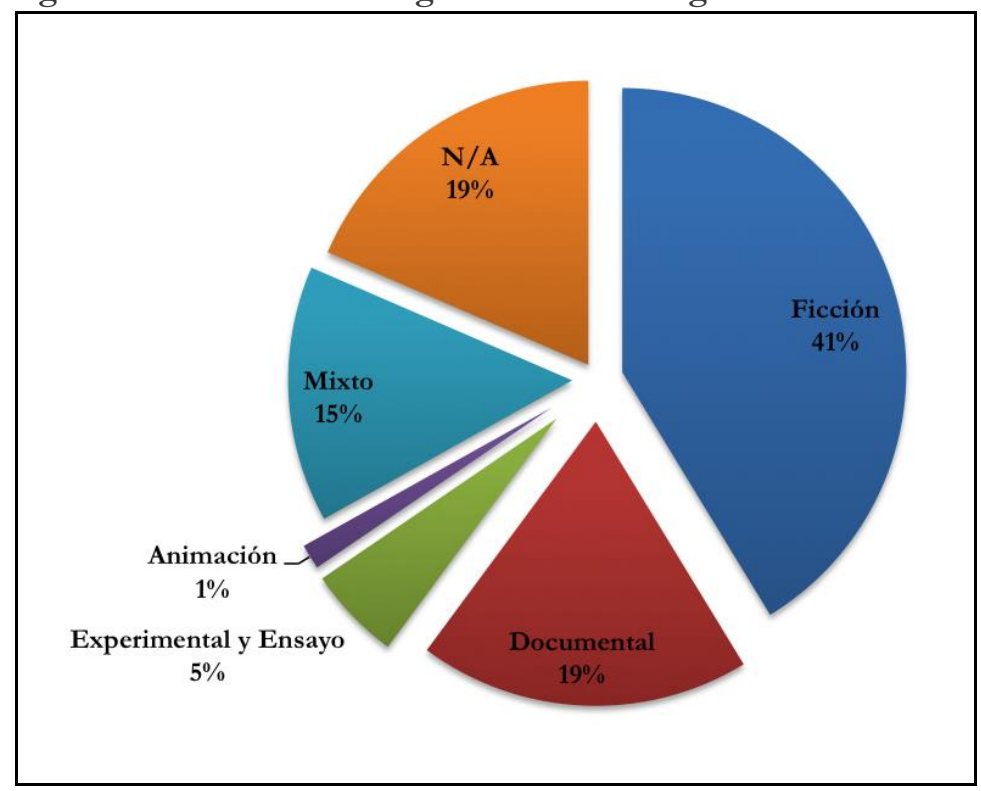




\subsubsection{Revisión: Temas y objetos de estudio}

El gráfico de la Figura 12 muestra el comportamiento de las tres categorías más recurrentes en Tema y objeto de estudio en los últimos 10 años: Realizadores, Identidad e Industria.

La categoría Realizadores (30\%) en los años 2010 y 2011 alcanza su frecuencia más alta, lo que se explica por la publicación del libro El cine de Raúl Ruiæ: fantasmas, simulacros y artificios (2010) y El novísimo cine chileno (2011), el primero con compilaciones de capítulos referidos al cineasta Raúl Ruiz y el segundo focaliza el estudio en realizadores de cine chileno contemporáneo.

La categoría Identidad (24\%) es la segunda más recurrente y su tendencia va en aumento desde el año 2011. Esta categoría está dividida en dos en la matriz de análisis: Identidad cultural, e Identidad y sujeto. La primera se observa más dominante con un $17 \%$, mientras que Identidad y sujeto $(6 \%)$ si bien es poco recurrente desde el 2005 hasta el 2011, demuestra ser un tema que va levemente en aumento a partir del año 2012, incremento que se debe a las publicaciones de los Encuentros de investigación sobre cine chileno y latinoamericano realizados en la Cineteca Nacional de Chile, y al libro Audiovisualy Política en Chile.

Industria es la tercera categoría más frecuente (20\%) concentrándose en los años 2012 y 2013 con la publicación de los libros Ya no basta con filmar. Las mejores conferencias de los primeros cuatro años de acción audiovisual (2012, varios autores), II Panorama del Audiovisual Chileno (2012, Valerio Fuenzalida y Pablo Julio, editores) y III Panorama del Audiovisual Chileno (2013, Valerio Fuenzalida y Johanna Wittle, editores).

La categoría Guión $(4,6 \%)$ se mantiene muy baja a lo largo de la década, salvo en el año 2008 en el cual se publican 10 libros de guiones de películas chilenas referidos en líneas precedentes. La categoría Intermedialidad/Intertextualidad (4\%) tiene un comportamiento bajo pero constante a partir del año 2011. La categoría Patrimonio Audiovisual (4\%), si bien se mantiene baja presenta una frecuencia mayor en el año 2011 con la publicación del libro El cine que fue: 100 años de cine chileno. Las categorías más bajas son Estudios Comparados (2\%) y Cine de regiones de Chile (1\%). 
Figura 12. Comportamiento de las categorías de Temas y objeto de estudio

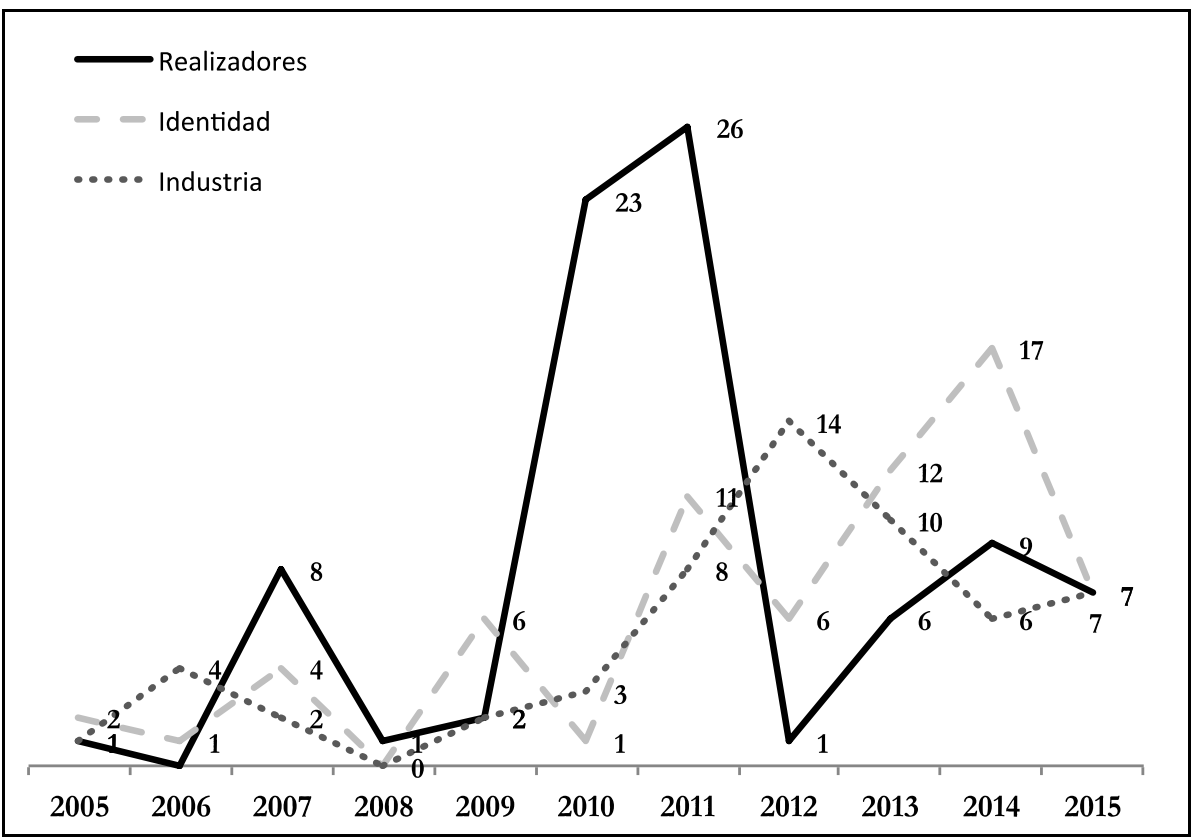

\subsubsection{Revisión: Tipo de investigación/análisis}

La mayoría de las publicaciones aborda un Análisis Cualitativo (80\%), lo que guarda relación con la aproximación disciplinaria, en la cual lideran las categorías de Ciencias Sociales (33\%), Estética (28\%) e Historia (15\%), mientras que los textos de Análisis Cuantitativo (9\%) se relacionan con la categoría de Economía de la cultura (13\%) como aproximación disciplinaria. Las investigaciones con Análisis Mixto (6\%) presentan asimismo mayor recurrencia con el acercamiento de Economía de la cultura.

\subsubsection{Revisión: Financiamiento de las publicaciones}

Un 48\% de las publicaciones de libros y capítulos de libros analizados indica la información de su financiamiento en la misma publicación. En los porcentajes que se consignan en este apartado y en relación a los capítulos de libro, se ha considerado para la distinción de financiamiento si el capítulo indica algún tipo 
de financiamiento en la misma publicación, o bien, si el libro total declara tener algún tipo de financiamiento como volumen total.

Del 48\% informado, el financiamiento por parte del Estado equivale al de mayor presencia $(64 \%)$ a través de diversas iniciativas de fomento: Fondo de Fomento Audiovisual (FFA), Fondo Nacional de Desarrollo y Tecnología (FONDECYT), Fondo Nacional de Fomento a la Lectura y el Libro (FNFLL), Consejo Nacional de la Cultura y las Artes (CNCA), Fondo Nacional para el Desarrollo Cultural y las Artes (FONDART), Fondo Nacional de Desarrollo Regional (FNDR) y Corporación de Fomento de la Producción (CORFO).

La categoría Mixta, que comprende Privado + Universidad y Estado + Universidad, ocupa el segundo lugar en financiamiento con un 21\%. En este categoría de financiamiento, están presentes la Pontificia Universidad Católica de Chile y la Universidad de Chile.

La categoría Universidad ocupa el tercer lugar en financiamiento para los estudios de cine chileno, con un escaso 9,6\%. Este porcentaje es compartido entre las siguientes universidades: Pontificia Universidad Católica de Chile, Universidad de Santiago de Chile, Universidad Alberto Hurtado y Universidad de Artes, Ciencias y Comunicación (UNIACC).

\section{Conclusiones}

Como se ha revisado, el panorama de campo de los estudios sobre cine chileno publicados en Chile demuestra un incremento sostenido desde el año 2010 al 2015, el que se observa se sostiene particularmente por la publicación de trabajos como capítulos de libros y artículos académicos.

En la década en estudio y considerando la escena total, entre los años 2005 y 2009 se registran 72 publicaciones entre libros, capítulos de libros y artículos en revistas académicas, mientras que entre el año 2010 y 2015 los resultados arrojan un total de 317 publicaciones. Este incremento del $440 \%$ en los últimos cinco años da cuenta de un movimiento relevante de reflexión del campo cultural. Los números son una señal de ello, aun cuando, evidentemente, no bastan para verificar la conformación o desfiguración de campo. 
En primer lugar, el análisis de la producción de textos publicados como libros y capítulos de libro junto a los intereses de investigación dominantes y recesivos en este corpus analizado, requiere ser completado con el análisis aún pendiente de los artículos académicos. En segundo lugar, se comprende que esta investigación en curso es una primera estación en el itinerario de observación de campo; estación que, habiéndola alcanzado, requeriría ser ampliada al análisis del posible diálogo que se establece entre los distintos estudios publicados y agentes involucrados. Sería importante, a su vez, incluir para el análisis en una siguiente investigación las tesis de postgrado, de modo de observar si existe un movimiento de reflexión de campo en esta esfera de estudios sobre cine en Chile.

Este proyecto aspira a ser un primer acercamiento metodológico al campo de estudio. La matriz final de análisis con la que se trabaja podrá parecer arbitraria -y en alguna medida lo es, cómo podría no serlo-. Con todo, luego de las rondas de consulta a expertos y expertas, y puesta a prueba de las distintas versiones del instrumento de análisis, se consideró que en las siete zonas finales de observación, con su consecuente despliegue de categorías y sub categorías, es posible distinguir cuestiones significativas para configurar una primera lectura del estado de campo de los estudios de cine en Chile.

Se concede que la matriz podrá ser juzgada como un instrumento tan cuidado como imperfecto, que generará tanto acuerdos como desacuerdos, y se reconoce que en ello radica precisamente su potencial. Los temas de discusión en la materia que nos convoca para este proyecto pueden ser ocasión de diálogo compartido y encuentro, también en las diferencias y la dificultad. La propia matriz fue ya un lugar de reflexión, un paso que aporta para la conformación de una comunidad de pensamiento acerca de pensar los estudios de cine.

Confirmar que los estudios de cine en Chile han tenido un crecimiento exponencial en los últimos años, es un indicador para construir la futura articulación del campo cultural a nivel nacional, reconociendo las áreas que predominan como intereses de investigación así como aquellas que están todavía ausentes. Este estudio permitirá asimismo reconocer aquellos temas que concentran el financiamiento estatal y universitario, así como aquellos que son abordados en forma autónoma por los investigadores y que se desarrollan vislumbrando la apertura del horizonte de investigación.

Una vez concluida esta investigación, los resultados finales arrojarán datos cuantitativos y cualitativos que aspiran a ser un aporte en la implementación de directrices de apoyo a nivel nacional para la investigación del cine chileno 
tanto en el ámbito académico como en el de las políticas estatales. A nivel latinoamericano, se proyecta que esta investigación sea un acercamiento a otros mapas de cine, métodos y resultados que se están desarrollando en los distintos países, estableciendo un diálogo de campo que posibilite articular proyectos de reflexión teórica en los que se vincule e impulse el estudio de nuestros cines.

El mapa no es el territorio y el camino recién se inicia. En este cometido, alienta tener la convicción de que se realiza una investigación inédita, que el intenso trabajo que se ha llevado a cabo desde enero del 2016 está ya arrojando los primeros resultados, y que esta investigación delineará un primer acercamiento a la observación del campo de los estudios de cine en Chile.

El equipo de investigación agradece, sinceramente, a todos y todas quienes participaron de nuestras consultas de matriz, y a todos y todas quienes han colaborado generosamente en el desarrollo de este proyecto. Las opiniones y sugerencias de todos ellos colaboran en la construcción de un diálogo especializado que cruce distintas zonas de experiencia y abra el campo a nuevos desafíos.

\section{Bibliografía}

Informe CAEM (2015). El cine en Chile en el 2015. Informe elaborado por la Cámara de Exhibidores Multisalas de Chile A.G. (CAEM). Recuperado de: http://www.caem.cl/index.php/informes-anuales/item/23-el-cine-en-chileen-el-2015 (consultado el 10 de abril de 2016).

Mouesca, J. y Orellana C. (1998). Cine y memoria del sigo XX. LOM ediciones, Santiago de Chile.

Parada, M. (2011). "El estado de los estudios sobre cine en Chile: una visión panorámica 1960-2009”. Razón y Palabra 77. México, agosto-octubre.

Recuperado de http://www.razonypalabra.org.mx/varia/77\%205a\%20parte/67_Parada_V77. pdf

Rojas, S. (2016). "Reflexión estética contemporánea. Entrevista de Alex Ibarra al filósofo Sergio Rojas". Le monde diplomatique. Edición chilena, del 20 de enero:

http://www.lemondediplomatique.cl/Reflexion-estetica-contemporanea.html 
Stange, H. y Salinas, C. (2008). "La incipiente literatura sobre cine chileno. Obra en construcción". La Fuga. Dossier Estados del Cine Chileno.

Recuperado de: http://www.lafuga.cl/la-incipiente-literatura-sobre-cinechileno/302 (consultado el 6 de agosto de 2009).

- (2009). "Hacia una elucidación del campo de estudios sobre cine en Chile". Aisthesis 46. Revista chilena de investigaciones estéticas, Instituto de Estética - Pontificia Universidad Católica de Chile, p.p. 270-283.

Trejo, R. (2009). Cine, Neoliberalismo y Cultura. Crítica a la economía política del cine chileno contemporáneo. Santiago, Chile: Arcis.

Zavala, L. (2009). "Los estudios sobre cine en México: Un terreno en construcción”. (Conferencia plenaria: Encuentro anual ASAECA, Asociación argentina de estudios de cine y audiovisual. 16-19 de junio). Tandil, Argentina. 


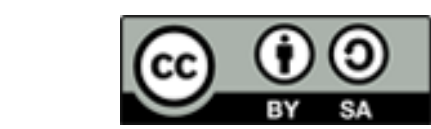

Licencia Creative Commons

Miguel Hernández Communication Journal

mhjournal.org

\section{Cómo citar este texto:}

María Marcela Parada Poblete (2017): “ Los estudios cinematográficos en Chile: aproximaciones a la conformación y reflexión de campo", en Miguel Hernández Communication Journal, nº, pp. 85 a 119. Universidad Miguel Hernández, UMH (ElcheAlicante). Recuperado el $\_$de de 20_ de: @ink del artículo en mhjournal.org] 
